\title{
Random gene dissection: a tool for the investigation of protein structural organization
}

\author{
Rimantas Sapranauskas and Arvydas Lubys
}

BioTechniques 39:395-402 (September 2005)

\begin{abstract}
To investigate the domain structure of proteins and the function of individual domains, proteins are usually subjected to limited proteolysis, followed by isolation of protein fragments and determination of their functions. We have developed an approach we call random gene dissection (RGD) for the identification of functional protein domains and their interdomain regions as well as their in vivo complementing fragments. The approach was tested on a two-domain protein, the type IIS restriction endonuclease BfiI. The collection of BfiI insertional mutants was screened for those that are endonucleolytically active and thus induce the SOS DNA repair response. Sixteen isolated mutants of the wild-type specificity contained insertions that were dispersed in a relatively large region of the target recognition domain. They split the gene into two complementing parts that separately were unable to induce the SOS DNA repair response. In contrast, all 19 mutants of relaxed specificity contained the cassette inserted into a very narrow interdomain region that connects BfiI domains responsible for DNA recognition and for cleavage. As expected, only the N-terminal fragment of $\mathrm{Bfi} I$ was required to induce SOS response. Our results demonstrate that RGD can be used as a general method to identify complementing fragments and functional domains in enzymes.
\end{abstract}

\section{INTRODUCTION}

Multifunctional proteins consist of two or more domains, each of which possess an autonomous structure and are associated with a particular function (1-4). It has been suggested that multidomain proteins have evolved by the fusion of two or more ancestral single domain proteins or that they are the result of recombination events such as domain duplication or swapping (5-8). New combinations of covalently joined domains are of great importance in protein evolution because they have a potential to ensure direct linkages between proteins that act in a coordinated manner, such as successive enzymes in the same metabolic pathway, or between proteins that belong to different functional systems, such as various signaling systems or transcriptional regulators. The repertoire of types of domains in proteins is limited $(9,10)$. Nevertheless, the huge diversity of proteins found in nature seems to stem from occasional recom- binational joining ("domain shuffling") followed by natural selection of the most effective multidomain variants (10-12).

Three-dimensional (3-D) structural data and biochemical properties of individual domains show that, in general, they are distinct, compact structural and functional units connected by flexible linkers. Usually, intradomain peptide units are either buried in the protein core or they comprise regular secondary structural elements. The sites of limited proteolysis are flexible or unfolded (13), and for that reason intradomain peptide units are less susceptible to proteases than interdomain regions, which usually form surface-exposed mobile loops. This protein architecture enables the use of limited proteolysis to elucidate the structural organization of multidomain proteins $(14,15)$. Protein fragments produced by this procedure are isolated and tested for their biochemical properties individually, a task that is sometimes complicated and labor intensive. Moreover, limited proteolysis may not be accurate in all cases. Given that modeling studies of proteolytic sites have revealed local unfolding of a stretch of up to 12 residues (16), correctly folded native proteins should be attacked by protease if they contain flexible sites in the protein chain that can bind and adapt to the specific stereochemistry of the protease active site (17). However, some short unfolded interdomain regions could be inaccessible to proteases. In addition, some loops could be resistant to proteolysis due to the absence of amino acid residues required for specific interaction with proteases.

Several computer algorithms were developed for the identification of domains of proteins for which 3-D structures are known (18-22). The algorithms are based on principles such as interface area minimization, distance mapping, identification of hydrophobic core, or domain compactness. Some of these algorithms are used in protein 
domain databases such as the Class Architecture Topology Homology (23) or Dali Domain Dictionary (24). Other methods for the prediction of domain boundaries rely on attempting to deduce homology-based domain boundaries via comparative sequence searches. However, this strategy can only be successful when the investigated sequence has detectable similarity to other sequence fragments in databases (25-30). Recently, a few novel methods have been developed to predict domain boundaries from amino acid sequence alone (31-34). Although computational methods for domain assignment are relatively accurate, all predictions must be confirmed experimentally.

Here we introduce random gene dissection (RGD), a technique that is designed to investigate the domain structure of proteins. This approach helps to overcome limitations of the proteolytic studies mentioned above, and it can be used to identify functional domains as well as interdomain regions and complementing protein fragments. The RGD approach is based on an idea that protein domains possessing autonomous structure are associated with a particular function that can be performed even in the absence of adjacent domains.

We used the RGD approach to study domains of the type IIS restriction endonuclease $B f i I$. This endonuclease recognizes the sequence 5'-ACTGGG$3^{\prime}$ and cleaves the upper DNA strand 5 nucleotides and the bottom DNA strand 4 nucleotides away from the recognition site (35) in the absence of $\mathrm{Mg}^{2+}$ (36). Bfil was shown to form a dimer in solution. Previous experiments have suggested that this enzyme is composed of two domains $(36,37)$ : an $\mathrm{N}$-terminal catalytic domain and a C-terminal domain responsible for target recognition. In the presence of the specific oligonucleotide, thermolysin selectively cleaved $B f i$ at Leu 191 or Leu193, resulting in two oligopeptides (38). After their separation, it was demonstrated that one of them is the $\mathrm{N}$-terminal domain, which is responsible for dimerization. In addition, this domain acts as a nonspecific nuclease. In contrast, the $\mathrm{C}$-terminal proteolytic fragment was able to bind the specific DNA, obviously indicating that it plays a role of DNA recognition domain (38).

In order to isolate catalytically active $B f i$ insertional mutants, we applied the genetic screening approach developed by Heitman et al. (39-41). In that system, intracellular double-stranded DNA cleavage, carried out by the restriction endonuclease EcoRI, results in the induction of genes involved in the SOS DNA repair response. It appears that DNA damage is the primary reason for the induced SOS response, which is responsible for DNA repair in the cell (42); thus, the phenotype of SOS induction has a potential to be applied in all experiments where intracellular endonucleolytic activity has to be identified. In the case of EcoRI, the SOS response was visualized by using Escherichia coli cells in which the SOS regulon gene $\operatorname{din} D 1$ was fused to the reporter gene lacZ. The development of blue color in these cells, when plated on media containing 5-bromo-4-chloro-3indolyl- $\beta$-D-galactopyranoside (X-gal), is therefore a measure of the SOS response. E. coli strains carrying the $\operatorname{din} D 1:: l a c Z$ gene fusion were used to isolate restriction endonuclease mutants of relaxed or altered substrate specificity $(40,43)$ as well as for the identification of transformants carrying the cloned restriction-modification genes (44). Combining this genetic screening and characterization method for active mutants of endonucleases with the RGD approach allowed us to predict boundaries of BfiI structural/ functional domains. Also, it helped us to isolate $B f i$ mutants with wild-type specificity that were encoded by two in vivo complementing oligopeptides.

\section{MATERIALS AND METHODS}

\section{Bacterial Strains and Media}

E. coli strain ER2267 was used as a host in subcloning procedures. Screening for endonucleolytically active BfiI mutants was done in ER1992 (44). Both strains were obtained from New England Biolabs (Beverly, MA, USA). RR1 (45) and XL1-Blue (46) strains were used to transfer genes for $B f i$ methyltransferases into the conjugative plasmid $\mathrm{F}^{\prime}$. The RR1-Bfi cells produced $B f i$ methyltransferases encoded by $\mathrm{F}^{\prime}:: \mathrm{mTn} 10$ (bfiIMC1 $1^{+}$ bfiIMC2 ${ }^{+}$). The construction of this strain is described in the Supplementary Methods available online at www. BioTechniques.com. All strains were grown in Luria-Bertani (LB) medium (47) at $37^{\circ} \mathrm{C}$. LB was supplemented with $1.5 \%$ agar for the plates. The following concentrations of antibiotics were used when necessary: ampicillin (Ap), $100 \mu \mathrm{g} / \mathrm{mL}$; kanamycin (Km), 50 $\mu \mathrm{g} / \mathrm{mL}$; chloramphenicol $(\mathrm{Cm}), 10 \mu \mathrm{g} /$ $\mathrm{mL}$; streptomycin $(\mathrm{Sm}), 30 \mu \mathrm{g} / \mathrm{mL}$; and tetracycline (Tc), $15 \mu \mathrm{g} / \mathrm{mL}$. X-gal was added to media at a final concentration of $50 \mu \mathrm{g} / \mathrm{mL}$. E. coli transformations were carried out either by electroporation or by $\mathrm{CaCl}_{2}$-heat shock method (47).

\section{Reagents, Oligonucleotides, and Enzymes}

All molecular biology kits and enzymes (except DNase I) were obtained from Fermentas UAB (Vilnius, Lithuania) and used as recommended by the manufacturer. DNase I was purchased from Sigma-Aldrich (St. Louis, MO, USA). Oligonucleotides were synthesized at Fermentas UAB. Sequencing primers were labeled with $\left[\gamma^{33} \mathrm{P}\right]$ ATP from Amersham Biosciences (Little Chalfont, UK).

\section{DNA Manipulations}

The construction and isolation of recombinant plasmids and other molecular manipulations followed standard protocols (47). A DNA Extraction Kit (Fermentas UAB) was used to purify DNA fragments from agarose gels. The precise mapping of the DNA cassette insertion point was done by the dideoxy nucleotide chain termination approach (48) using the Cycle Reader ${ }^{\mathrm{TM}}$ DNA Sequencing Kit (Fermentas UAB) and a pair of outward primers complementary to both ends of the cassette: $5^{\prime}$ GTTCTTTACGATGCCATTGGG-3' and 5'-GTGATGGCTTCCATGTCGGC$3^{\prime}$. Plasmids used in this study and their constructs are described online in Supplementary Table S1. Random gene dissection of the $B f i$ I restriction enzyme was done using a technique 
similar to that described by Biondi et al. (49). Fifty micrograms of pUCBfiIR DNA and the same amount of ethidium bromide were diluted in $1 \mathrm{~mL}$ of $\mathrm{G}^{+}$ buffer (Fermentas UAB). In a control reaction, $50 \mu \mathrm{L}$ of this solution were incubated with $100 \mathrm{pg}$ DNase I. Aliquots of control reaction were

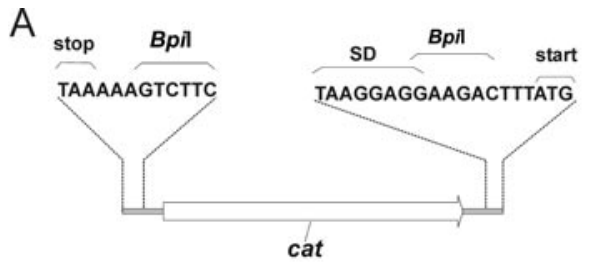

B

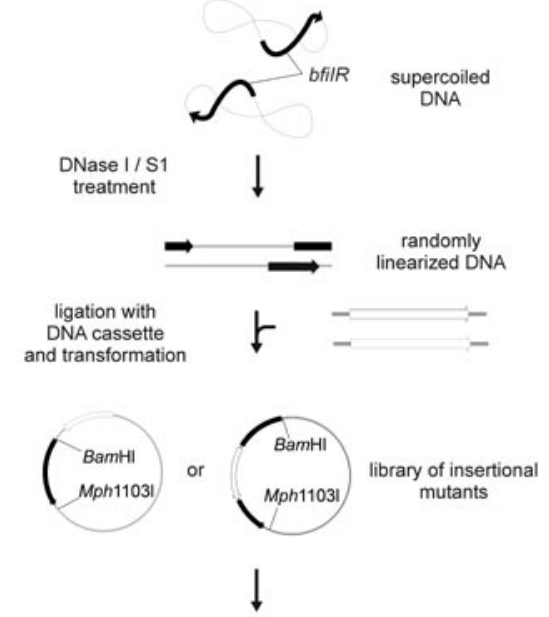

digestion of total plasmid DNA with BamHI-Mph1103I, isolation of bfilR genes carrying the inserted DNA cassette, ligation with vector backbone

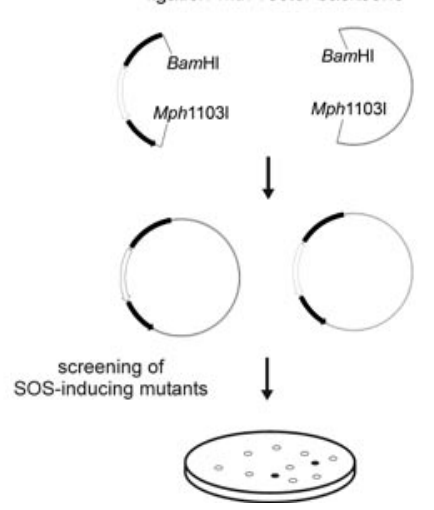

Figure 1. Construction of random insertions. (A) The structure of the cassette used for random gene dissection. The locations of the translation termination codon (stop), the Shine-Dalgarno sequence (SD), and translation initiation (start) codon are shown. The blunt-ended DNA cassette was prepared by cleavage of pUCm with the type IIS restriction endonuclease BpiI and by filling in using T4 DNA polymerase. Resistance to chloramphenicol (cat gene) was used to select recombinant plasmids. (B) Experimental overview. removed at different time points and analyzed to evaluate the kinetics of the appearance of open circular plasmids. We observed that incubation for $5 \mathrm{~min}$ at $30^{\circ} \mathrm{C}$ results in $90 \%$ of nicked DNA. Therefore, $1.9 \mathrm{ng}$ DNase I were added to the remaining DNA solution, and the reaction was terminated after $5 \mathrm{~min}$ of incubation at $30^{\circ} \mathrm{C}$ by adding EDTA to a final concentration of $50 \mathrm{mM}$. Nicked circular plasmids were gel-purified on $0.8 \%$ agarose and treated with $\mathrm{S} 1$ nuclease in S1 buffer (Fermentas UAB) to cut the second DNA strand at the position of the nick. The incubation time with the S1 nuclease was chosen to convert only about $40 \%$ of nicked DNA into the linear form to avoid degradation of linear DNA ends as much as possible. These randomly linearized plasmids were gel-purified and dephosphorylated using calf intestine alkaline phosphatase. The plasmids were ligated with a specially designed cassette coding for chloramphenicol resistance, which was excised from pUCm with BpiI and then blunted using T4 DNA polymerase. The ligation mixture was used to transform electrocompetent RR1-Bfi cells. Transformants were selected on LB agar supplemented with Ap, Cm, and isopropyl- $\beta$-D-thiogalactoside (IPTG; $1 \mathrm{mM}$ ). Plasmids isolated from the pooled transformants contained the cassette inserted either within the restriction endonuclease gene or in other transcriptionally active locations (for instance, upstream or downstream of the gene for BfiI). The latter group of mutants was removed from the collection by an enrichment procedure based on subcloning only those mutant genes in which the insertion occurred within the gene. In pUCBfiIR, BamHI and $M p h 1103$ I have unique cleavage sites located on both ends of bfilR. Those sites are $1.15 \mathrm{~kb}$ apart. When the total plasmid DNA of pUCBfiIR insertional mutants was cleaved with a mixture of BamHI and Mph1103I, a DNA fragment of $1.85 \mathrm{~kb}$ appeared $(1.15 \mathrm{~kb}$ and the DNA cassette of 0.7 $\mathrm{kb})$. The fragment was gel-purified and ligated with a pUCBfiIR backbone $(2.7 \mathrm{~kb})$ that had been produced by digestion with BamHI and Mph1103I. The ligation mixture was introduced into RR1-Bfi cells by electroporation.
Plasmid DNA was isolated from the pooled $\mathrm{Ap}^{\mathrm{r}} \mathrm{Cm}^{\mathrm{r}}$ transformants and used for the isolation of endonucleolytically active $B f i$ mutants.

\section{Screening for Functionally Active bfiIR Mutants and Their Deletion Analysis}

The genetic screening technique $(39,40)$ was used to isolate functionally active BfiI mutants in E. coli strain ER1992, which carries the dinD1::lacZ fusion. The colonies of ER1992 with induced SOS DNA repair response can be identified by their blue color on LB agar supplemented with chromogenic LacZ substrate X-gal (44). The ER1992 strain was used to screen for $B f i \mathrm{I}$ mutants that are able to induce the SOS DNA repair response in the absence of protective methylation. In parallel, those BfiI mutants that induced the SOS response in the presence of cognate methylation were identified using $B f i \mathrm{I}$ methylation-proficient ER1992 derivative ER1992 (pACBfiIM).

Gene fragments coding for either the $\mathrm{N}$-terminal or C-terminal oligopeptides of the $B f i$ insertional mutants were deleted to define their importance in the SOS induction. These deletions were generated by the removal of the corresponding DNA region flanked by either an $N c o \mathrm{I} / P s t \mathrm{I}$ cleavage site or an $N c o \mathrm{I} / E c l 136 \mathrm{II}$ site. The $N c o \mathrm{I}$ site is located within the cassette, whereas the PstI and Ecl136II sites are in the pUC57 multiple cloning site. The resulting deletion derivatives were transformed back into ER1992 or ER1992 (pACBfiIM) and their capability to induce the SOS DNA repair response was evaluated.

\section{RESULTS}

\section{Design of the Cassette for Random Gene Dissection}

The RGD technique (Figure $1 B$ ) is based on the use of specially designed DNA cassette (Figure 1A). This cassette, when properly inserted into the reading frame of a target gene, results in the premature termination of translation of its messenger RNA (mRNA) due to the presence 
of a TAA stop codon at the $5^{\prime}$ end of the cassette. Because the $3^{\prime}$ end of the cassette contains a ribosome binding site followed by the initiation codon ATG, the remainder of the target gene is translated as a separate polypeptide. Such a construct encodes pieces of the target protein without any additional amino acids except for the initiator formyl-methionine. The presence of the cat gene for chloramphenicol resistance in the cassette facilitates selection for insertional mutants because the cat gene has no promoter of its own. This gene can be transcribed only if the cassette is correctly inserted into a transcriptionally active DNA region. Of note, the level of antibiotic resistance of cells harboring the appropriate resistance gene depends on its expression level. In order to determine the $\mathrm{Cm}$ concentration that could be tolerated by cells carrying the cassette under the control of pUC57 promoter $\mathrm{P}_{\text {lac }}$, the cassette was inserted into the Eco32I site of this plasmid. We observed that the appearance of chloramphenicolresistant transformants of normal size required $\mathrm{P}_{\text {lac }}$ induction with $1 \mathrm{mM}$ IPTG and lowering the $\mathrm{Cm}$ concentration to $10 \mu \mathrm{g} / \mathrm{mL}$. These conditions were used throughout this study to create and analyze the library of bfiIR insertional mutants.

\section{Random Dissection of the bfiIR Gene}

Single-stranded nicks were introduced into supercoiled pUCBfiIR, in which the gene for $B f i \mathrm{I}$ restriction endonuclease is under the control of $\mathrm{P}_{\text {lac }}$ by incubating plasmid DNA with DNase $I$ in the presence of $\mathrm{Mg}^{2+}$ and ethidium bromide. In the next step, these nicks were converted into doublestranded breaks by the use of nuclease S1. The resulting linear molecules of near to full-length size were gel-purified and ligated with the blunt-ended DNA cassette. The ligation mixture was introduced into $E$. coli cells expressing the $B f i$ methyltransferases, yielding $10^{5}$ $\mathrm{Cm}$-resistant colonies. Noteworthily, the size of individual colonies varied, indicating either the toxicity of some mutations or differences in the individual levels of chloramphenicol resistance, which most likely depended

Table 1. The Structure of Bfil Mutants of Wild-Type Specificity

\begin{tabular}{|lll|}
\hline Mutant $^{\mathbf{a}}$ & $\begin{array}{l}\text { N-Terminal } \\
\text { Oligopeptide }\end{array}$ & $\begin{array}{l}\text { C-Terminal } \\
\text { Oligopeptide }\end{array}$ \\
\hline WTS31 & $1-217$ & $218-358$ \\
WTS37 & $1-219$ & $220-358$ \\
WTS39 & $1-220$ & $221-358$ \\
WTS5 & $1-248$ & $249-358$ \\
WTS29 & $1-263$ & $267-358$ \\
WTS25 & $1-263$ & $269-358$ \\
WTS27 (WTS7) & $1-264$ & $266-358$ \\
WTS23 (WTS33) & $1-264$ & $267-358$ \\
WTS3 & $1-264$ (+230) & $269-358$ \\
WTS17 (WTS21) & $1-265$ & $268-358$ \\
WTS19 & $1-266$ & $268-358$ \\
WTS1 & $1-267$ & $269-358$ \\
WTS11 & $1-270$ & $271-358$ \\
Numbers in columns refer to the first and the last amino acid residue of the respective oligopeptide \\
relative to the amino acid sequence of the wild-type Bfil. \\
anutants that have identical structure are indicated in parentheses. \\
bThe number in parenthesis indicates the number of amino acid residues derived from the inserted \\
cassette.
\end{tabular}

on the position of the inserted cassette. Restriction mapping of the total plasmid DNA isolated from pooled transformants indicated that about $40 \%$ of all insertions occurred outside of the restriction endonuclease gene. These were eliminated by selecting only the DNA fragments of a size matching the bfilR gene with the inserted cassette, subcloning these fragments into the appropriately cleaved pUCBfiIR, and then selecting for the $\mathrm{Cm}$-resistant transformants.

\section{Isolation of $B f i$ I Mutants with the Wild-Type Specificity}

To screen for mutants with the wildtype substrate specificity, an enriched library of the BfiI insertional mutants was introduced into ER1992. The cells were plated onto LB agar supplemented with X-gal and screened for the relatively abundant (2\%) dark-blue colonies. Eighteen dark-blue colonies were selected for further analysis. Individual plasmid DNAs isolated from the selected colonies were introduced into both ER1992 and ER1992 (pACBfiIM) in order to evaluate the specificity of mutant variants of the endonuclease. ER1992 (pACBfiIM) is a derivative of ER1992 that produces $B f i \mathrm{I}$ methyltransferases and thus tolerates the presence of wild-type $B f i$ restriction endonuclease. It was expected that enzymes with the wild-type specificity would be unable to induce the SOS DNA repair response in ER1992 (pACBfiIM), while the induction of the SOS response by relaxed-specificity mutants was expected to be independent of the DNA methylation. One plasmid was discarded because it did not induce the SOS response in any strain. Of the remaining 17 plasmids, 16 induced the SOS response in ER1992, but not in ER1992 (pACBfiIM). These insertional mutants with the wild-type specificity of the endonuclease were abbreviated as WTS (Table 1). The endonuclease activity of these mutants seemed to be low because the recipient cells survived even in the absence of the protective effect of DNA methylation. One mutant, REL15, induced the SOS response in both methyltransferase-minus and methyltransferase-plus strains, suggesting that the insertion relaxes the specificity of BfiI (REL stands for relaxed specificity). This mutant plasmid was extremely toxic to recipient cells. The sequence analysis revealed that in REL15, the cassette terminated the translation of the protein at the putative linker region that joins separate $B f i$ d domains that are responsible for DNA cleavage and for 
target recognition (Table 2). Further deletion analysis showed that only the $\mathrm{N}$-terminal fragment of REL15 was required to induce the SOS response (Figure 2). Surprisingly, the orientation of the cassette in REL15 was found to be inverted as compared with other mutants. This finding suggests that an $E$. coli promoter-like structure is located within the noncoding strand of the $b f i I R$ gene. Alternatively, it could have formed accidentally after the insertion of the cassette. Sequencing of the other mutants (Table 1) allowed us to pinpoint three regions of the endonuclease where disruption of the enzyme was possible. The largest one was the region between $\mathrm{Asp}^{263}$ and $\mathrm{Gln}^{270}$, in which a deletion of up to 5 amino acid residues was possible (mutant WTS25). The second region was delimited by $\mathrm{Pro}^{217}$ and $\mathrm{Thr}^{220}$ (mutants WTS31, WTS37, and WTS39), and the third one was between Gly ${ }^{248}$ and $\mathrm{Thr}^{249}$ (mutant WTS5). To further characterize the endonuclease, DNA fragments coding for either the N-terminal or the C-terminal oligopeptides were then removed from mutants WTS37, WTS5, and WTS27. These deletion variants were tested for their ability to induce the SOS DNA repair response (Figure 2). The response was induced only if both oligopeptides were encoded,

\begin{tabular}{|c|c|c|c|}
\hline \multirow{3}{*}{ Mutant } & \multicolumn{3}{|c|}{ SOS response } \\
\hline & & $\mathrm{M}^{+}$ & $M^{-}$ \\
\hline & & - & 0 \\
\hline REL15， REL17， REL21 & $\nabla$ & + & + \\
\hline N-REL15, N-REL17, N-REL21 & 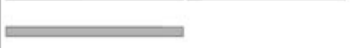 & + & + \\
\hline C-REL15, C-REL17, C-REL21 & $\nabla$ & - & - \\
\hline $\begin{array}{l}\text { WTS37 } \\
\text { N-WTS37 }\end{array}$ & 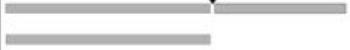 & $\overline{-}$ & $\begin{array}{l}+ \\
-\end{array}$ \\
\hline C-WTS37 & & - & - \\
\hline WTS5 & $\nabla$ & - & + \\
\hline N-WTS5 & 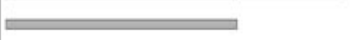 & - & - \\
\hline C-WTS5 & & - & - \\
\hline WTS27 & $\nabla$ & - & + \\
\hline N-WTS27 & 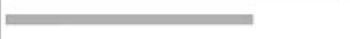 & - & - \\
\hline C-WTS27 & $=$ & - & - \\
\hline
\end{tabular}

Figure 2. Deletion analysis of isolated bfiIR insertional mutants. bfiIR mutants of relaxed substrate specificity (REL15, REL17, and REL21) and of wild-type specificity (WTS37, WTS5, and WTS27) were examined. Black triangles represent dissection points. Deletion derivatives were constructed as described in the Materials and Methods section. Gene regions coding for Nor C-terminal polypeptides were deleted in order to determine their involvement in the induction of SOS DNA repair response in the presence $\left(\mathrm{M}^{+}\right)$or in absence $\left(\mathrm{M}^{-}\right)$of cognate methylation. + , SOS DNA repair response detected [blue-colored colonies on Luria-Bertani (LB) agar plates containing 5-bromo-4-chloro-3-indolyl- $\beta$-D-galactopyranoside (X-gal)]; -, no SOS response; 0 , no transformants obtained. suggesting that the functional activity

\section{Isolation of $B f i I$ Mutants of Relaxed}

To screen for mutants with relaxed substrate specificity, a collection of $B f i \mathrm{I}$ insertional mutants was introduced into ER1992 (pACBfiIM). Transformation ed $0.2 \%$ blue colonies. Plasmids of the relaxed speciSuencing of the gene dissection point has revealed that insertions were lustered in a very narrow region of the polypeptide chain (Table 2). In total, Anas of their DNA sequences indicated that $\mathrm{N}$-terminal oligopeptides mutants were terminated at the derived from the cassette. the putative translation initiation codon of the C-terminal oligopeptide in four isolated mutants varied due to small fhis resulted in the out-ofposition of the cassette-encoded ATG (Table 2). Deletion analysis of two mutants, REL17 and REL21 (Figure 2 ), revealed that the N-terminal oligopeptide was sufficient to induce the SOS response. In order to test if both parts of the interrupted $B f i$ mutants are expressed, we analyzed the REL17 expression products by Western blot analysis using BfiIspecific antibodies. Two bands corresponding to the $\mathrm{N}$-terminal and the C-terminal fragments were identified (data not shown), suggesting that these cells tolerate the simul- taneous presence of both oligopeptides.

\section{DISCUSSION}

We developed a new protein fragmentation technique, which we call random gene dissection, in which a two-domain protein is dissected into two individual domains. This technique is based on a random DNase I/S1-mediated disruption of a gene followed by the insertion of a specially designed cassette. The cassette, when inserted in-frame, ensures a premature termination of the protein to produce an $\mathrm{N}$-terminal protein fragment and an independently translated C-terminal fragment. The well-studied restriction endonuclease $B f i \mathrm{I}$ was chosen as a model for proof-of-principle experiments.

It should be noted, however, that there are preferred cleavage sites for DNase I $(47,50)$. As a result, there is some bias regarding cassette insertion into preferred regions. To enhance the probability of targeting poor DNase I cleavage sites, we increased the size of the library of insertional mutants. This library was screened for endonucleolytically active $B f i$ I mutants both in the presence and in the absence of protecting methylation.

In the presence of site-specific BfiI methylation, host DNA can be cleaved (and the SOS response induced) only if the specificity of $B f i I$ was altered or the enzyme was converted into nonspecific nuclease. Therefore, only the mutants of relaxed specificity are expected in this screen. A set of 18 such mutants was analyzed thoroughly. In all of them, translation of the $\mathrm{N}$-terminal fragment was terminated within the narrow region of $B f i$ I starting with amino acid residue Asp ${ }^{185}$ and ending with $\mathrm{Thr}^{204}$. The deletion of gene fragments coding for C-terminal oligopeptides had no effect on the ability of mutants to induce the SOS DNA repair response, suggesting that the N-terminal oligopeptide possesses endonucleolytic activity. This is in agreement with the previous observation that the $\mathrm{N}$ terminal part of $B f i I$ acts as a nonspecific nuclease (38). Based on the results of our experiments, we also predict 
Table 2. The Structure of Bfil Mutants of Relaxed Specificity

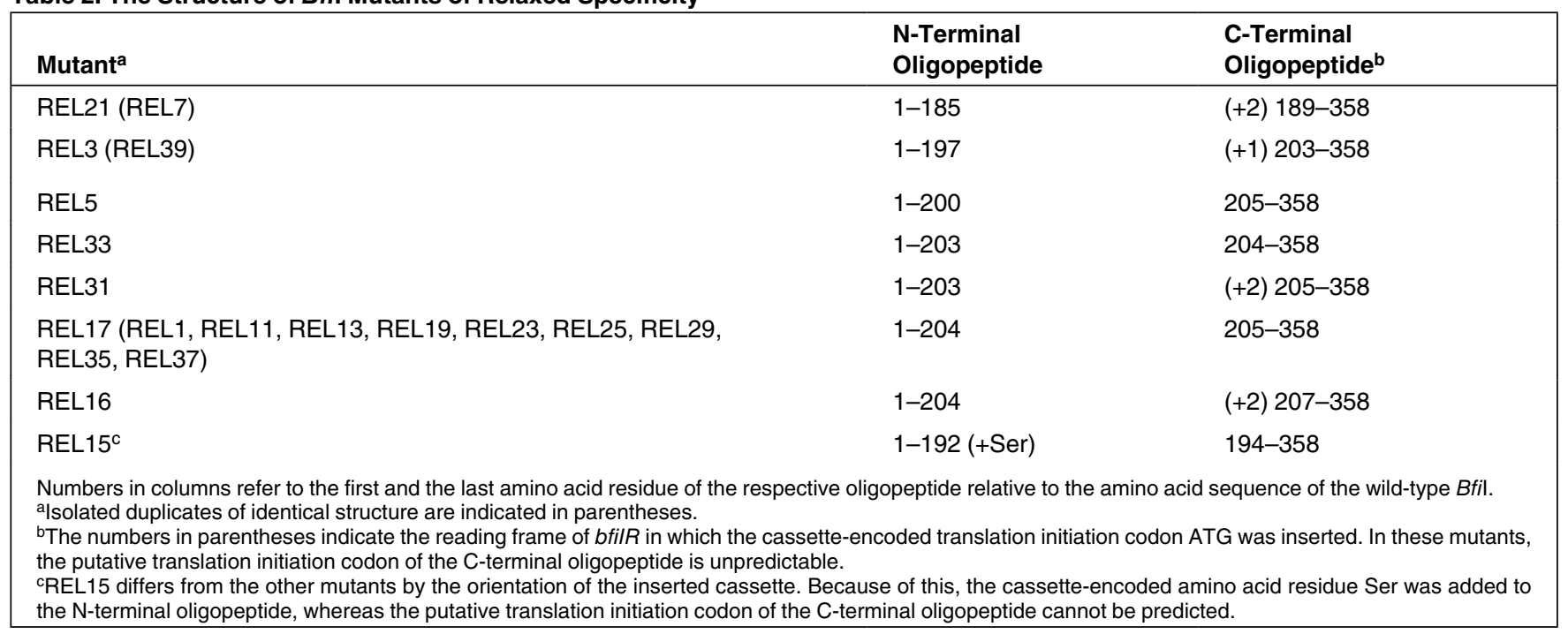

that a connecting linker between the $\mathrm{N}$-terminal and the $\mathrm{C}$-terminal domains extends from $\mathrm{Asp}^{185}$ to $\mathrm{Thr}^{204}$.

In the absence of $B f i$ I-specific methylation, insertional mutants of both types (i.e., those that cleave DNA specifically and those that hydrolyze DNA nonspecifically) should be able to induce the SOS response. Thus, both types of mutants have equal chances to be isolated in this screen. Of 17 mutants analyzed, however, only one was of relaxed specificity, whereas 16 mutants possessed the wild-type specificity. This fact indicates that either mutants of relaxed specificity are more detrimental to the host and only a small fraction of less toxic mutants survives, or there are more regions within $B$ fiI where splitting into two fragments without the loss of the wild-type specificity is possible. We found that both parts of the wild-type specificity mutants were required to induce the SOS response, indicating complementation of the $\mathrm{N}$ terminus and $\mathrm{C}$ terminus. Sequencing of these mutants revealed their cut points and allowed us to assign the mutants into three groups based on BfiI dissection region: $(i)$ between amino acid residues Pro $^{217}$ and Ala ${ }^{221}$; (ii) between amino acid residues $\mathrm{Gly}^{248}$ and $\mathrm{Thr}^{249}$; and (iii) between amino acid residues $\mathrm{Asp}^{263}$ and $\mathrm{Cys}^{271}$ (deletions of up to five amino acid residues were allowed within this region without the loss of function). A strong grouping of mutants suggests that BfiI dissection occurred in regions that tolerate those mutations. Of note, the third region can accept additional sequences (see Table 1, mutant WTS3). Therefore, it seems that cut points, at least in this region, are located within the surfacelocated protein secondary structure, most probably a loop. However, X-ray studies of BfiI crystals are necessary to confirm these predictions.

The RGD technique also allowed precise mapping of the BfiI interdomain linker. Its position perfectly matches the position identified by the limited proteolysis (38). This observation implies that RGD has a potential to replace limited proteolysis in experiments aimed to investigate the domain structure of proteins. This approach does not depend on the primary structure of the linker (i.e., the presence or absence of protease targets), and it does not require highly purified protein preparations. Moreover, RGD does not require structural information to convert monomeric enzyme into two complementing oligopeptides.

Overall, RGD has a potential to replace the incremental truncation approach when genes are shortened from either the $5^{\prime}$ or the $3^{\prime}$ terminus to produce collections of deletion derivatives, which are then combined in order to try to identify complementing pairs (51). Incremental truncation results in overlapping gene fragments that can recombine in vivo and restore the functional activity of the gene, resulting in false positives. This is not an issue for the RGD technique because individual fragments of the interrupted gene have no overlapping regions and thus cannot recombine.

Another advantage of RGD is that a limited number of the mutants needs to be analyzed because each mutant represents a unique bisection point. For example, a collection of 900 insertional mutants is adequate to create a collection of all theoretically possible insertional mutants of a 900-nucleotide gene. If combinatorial engineering is applied for the same gene, a significantly larger number of mutant combinations has to be analyzed $(900 \times$ $900=810,000)$. If both libraries are combined with 5-fold redundancy, then $2 \times 10^{7}$ transformants $(5 \times 900 \times 5 \times$ 900) need to be screened. Such a task requires the application of powerful selection techniques that are not always available. Using RGD, some level of redundancy is required to overcome both the preference of DNase I for some specific sequences and the tendency to form small deletions at the cassette insertion point (49). However, only $1.8 \times 10^{4}$ mutants are required to be produced and analyzed by RGD at a 20-fold redundancy. This number is 1000 times lower as compared with the number of variants generated by incremental truncations. Furthermore, each of $1.8 \times 10^{4}$ mutants can be individually screened for functional activity. Finally, each possible cut point is represented by only very few mutants in RGD, while the use of 
incrementally truncated proteins may result in multiple complementing pairs that are in fact variants of the same cut point. On the other hand, it appears that sometimes protein overlaps might be required for efficient complementation (52). If so, RGD will not identify those overlaps.

Taken together, we envision that the RGD technique will be used in experiments designed to isolate complementing oligopeptides and as an alternative to limited proteolysis in the study of enzyme domain structure. Also, RGD could prove to be a powerful technique in experiments aimed at identifying exact boundaries of individual domains in multidomain proteins of prokaryotes and eukaryotes. This information may help to express individual domains in soluble form, a task that is one of the main objectives of structural proteomics.

\section{ACKNOWLEDGMENTS}

We are grateful to Fermentas $U A B$ for providing the enzymes and kits. We wish to thank A. Janulaitis for helpful discussions during the work and the preparation of this manuscript, L. Grinius for comments and valuable improvements of the manuscript, E. Cesnaviciene for linguistic help, and $M$. Leckiene for her help during the development of the BfiI-specific antibodies.

\section{COMPETING INTERESTS STATEMENT}

A.L. is employed by Fermentas $U A B$, the manufacturer of some materials used in this study. R.S. declares no competing interests.

\section{REFERENCES}

1.Ritco-Vonsovici, M., B. Mouratou, P. Minard, M. Desmadril, J.M. Yon, M. Andrieux, E. Leroy, and E. Guittet. 1995. Role of the C-terminal helix in the folding and stability of yeast phosphoglycerate kinase. Biochemistry 34:833-841.

2.Minard, P., L. Hall, J.M. Betton, D. Missiakas, and J.M. Yon. 1989. Efficient expression and characterization of isolated structural domains of yeast phosphoglycerate kinase generated by site-directed mutagenesis. Protein Eng. 3:55-60.

3.Markovic-Housley, Z., A. Cooper, A. Lustig, K. Flukiger, B. Stolz, and B. Erni. 1994.
Independent folding of the domains in the hydrophilic subunit IIABman of the mannose transporter of Escherichia coli. Biochemistry 33:10977-10984.

4.Nock, S., N. Grillenbeck, M.R. Ahmadian, S. Ribeiro, R. Kreutzer, and M. Sprinzl. 1995. Properties of isolated domains of the elongation factor Tu from Thermus thermophilus HB8. Eur. J. Biochem. 234:132-139.

5.Gilbert, W. 1978. Why genes in pieces? Nature 271:501.

6.de Chateau, M. and L. Bjorck. 1996. Identification of interdomain sequences promoting the intronless evolution of a bacterial protein family. Proc. Natl. Acad. Sci. USA 93:8490-8495.

7.Yanai, I., A. Derti, and C. DeLisi. 2001. Genes linked by fusion events are generally of the same functional category: a systematic analysis of 30 microbial genomes. Proc. Natl. Acad. Sci. USA 98:7940-7945.

8.Teichmann, S.A., J. Park, and C. Chothia. 1998. Structural assignments to the Mycoplasma genitalium proteins show extensive gene duplications and domain rearrangements. Proc. Natl. Acad. Sci. USA 95:14658-14663.

9.Wolf, Y.I., N.V. Grishin, and E.V. Koonin. 2000. Estimating the number of protein folds and families from complete genome data. J. Mol. Biol. 299:897-905.

10.Koonin, E.V., Y.I. Wolf, and G.P. Karev. 2002. The structure of the protein universe and genome evolution. Nature 420:218-223.

11.Yanai, I., Y.I. Wolf, and E.V. Koonin. 2002. Evolution of gene fusions: horizontal transfer versus independent events. Genome Biol. 3: research0024.

12.Apic, G., J. Gough, and S.A. Teichmann. 2001. An insight into domain combinations. Bioinformatics 17:83-89.

13.Fontana, A., G. Fassina, C. Vita, D. Dalzoppo, M. Zamai, and M. Zambonin. 1986. Correlation between sites of limited proteolysis and segmental mobility in thermolysin. Biochemistry 25:1847-1851.

14.Hubbard, S.J. 1998. The structural aspects of limited proteolysis of native proteins. Biochim. Biophys. Acta 1382:191-206.

15.Fontana, A., P.P. de Laureto, B. Spolaore, E. Frare, P. Picotti, and M. Zambonin. 2004. Probing protein structure by limited proteolysis. Acta Biochim. Pol. 51:299-321.

16.Hubbard, S.J., F. Eisenmenger, and J.M. Thornton. 1994. Modeling studies of the change in conformation required for cleavage of limited proteolytic sites. Protein Sci. 3:757768.

17.Vita, C., D. Dalzoppo, and A. Fontana. 1985. Limited proteolysis of thermolysin by subtilisin: isolation and characterization of a partially active enzyme derivative. Biochemistry 24:1798-1806.

18.Rose, G.D. 1979. Hierarchic organization of domains in globular proteins. J. Mol. Biol. 134:447-470.

19.Zehfus, M.H. and G.D. Rose. 1986. Compact units in proteins. Biochemistry 25:5759-5765.

20.Holm, L. and C. Sander. 1994. Parser for protein folding units. Proteins 19:256-268.

21.Swindells, M.B. 1995. A procedure for detecting structural domains in proteins. Protein Sci. 4:103-112.

22.Siddiqui, A.S. and G.J. Barton. 1995. Continuous and discontinuous domains: an algorithm for the automatic generation of re- liable protein domain definitions. Protein Sci 4:872-884.

23.Pearl, F., A.Todd, I. Sillitoe, M. Dibley, O. Redfern, T. Lewis, C. Bennett, R. Marsden, et al. 2005. The CATH Domain Structure Database and related resources Gene3D and DHS provide comprehensive domain family information for genome analysis. Nucleic Acids Res. 33:247-251.

24.Dietmann, S. and L. Holm. 2001 Identification of homology in protein structure classification. Nat. Struct. Biol. 8:953-957.

25.Sonnhammer, E.L. and D. Kahn. 1994. Modular arrangement of proteins as inferred from analysis of homology. Protein Sci. 3:482492.

26.Adams, R.M., S. Das, and T.F. Smith. 1996. Multiple domain protein diagnostic patterns. Protein Sci. 5:1240-1249.

27.Park, J. and S.A. Teichmann. 1998 DIVCLUS: an automatic method in the GEANFAMMER package that finds homologous domains in single- and multi-domain proteins. Bioinformatics 14:144-150.

28.Gracy, J. and P. Argos. 1998. Automated protein sequence database classification. II. Delineation of domain boundaries from sequence similarities. Bioinformatics 14:174187.

29.Guan, X. and L. Du. 1998. Domain identification by clustering sequence alignments. Bioinformatics 14:783-788.

30.Gouzy, J., F. Corpet, and D. Kahn. 1999 Whole genome protein domain analysis using a new method for domain clustering. Comput. Chem. 23:333-340.

31.Wheelan, S.J., A. Marchler-Bauer, and S.H. Bryant. 2000. Domain size distributions can predict domain boundaries. Bioinformatics 16:613-618

32.George, R.A. and J. Heringa. 2002 SnapDRAGON: a method to delineate protein structural domains from sequence data. J. Mol. Biol. 316:839-851.

33.Marsden, R.L., L.J. McGuffin, and D.T. Jones. 2002. Rapid protein domain assignment from amino acid sequence using predicted secondary structure. Protein Sci. 11:2814-2824.

34.Liu, J. and B. Rost. 2004. Sequence-based prediction of protein domains. Nucleic Acids Res. 32:3522-3530

35.Vitkute, J., Z. Maneliene, M. Petrusyte, and A. Janulaitis. 1998. BfiI, a restriction endonuclease from Bacillus firmus S8120, which recognizes the novel non-palindromic sequence 5'-ACTGGG(N)5/4-3'. Nucleic Acids Res. 26:3348-3349.

36.Sapranauskas, R., G. Sasnauskas, A Lagunavicius, G. Vilkaitis, A. Lubys, and V. Siksnys. 2000. Novel subtype of type IIs restriction enzymes. BfiI endonuclease exhibits similarities to the EDTA-resistant nuclease Nuc of Salmonella typhimurium. J. Biol. Chem. 275:30878-30885.

37.Lagunavicius, A., G. Sasnauskas, S.E. Halford, and V. Siksnys. 2003. The metal-independent type IIs restriction enzyme BfiI is a dimer that binds two DNA sites but has only one catalytic centre. J. Mol. Biol. 326:10511064.

38.Zaremba, M., C. Urbanke, S.E. Halford, and V. Siksnys. 2004. Generation of the Bfil restriction endonuclease from the fusion of a DNA recognition domain to a non-specific nuclease from the phospholipase D superfamily. J. Mol. Biol. 336:81-92. 
39.Heitman, J., N.D. Zinder, and P. Model. 1989. Repair of the Escherichia coli chromosome after in vivo scission by the EcoRI endonuclease. Proc. Natl. Acad. Sci. USA 86:2281-2285.

40.Heitman, J. and P. Model. 1990. Mutants of the EcoRI endonuclease with promiscuous substrate specificity implicate residues involved in substrate recognition. EMBO J. 10:3369-3378.

41.Heitman, J. and P. Model. 1991. SOS induction as an in vivo assay of enzyme-DNA interactions. Gene 103:1-9.

42.Janion, C. 2001. Some aspects of the SOS response system - a critical survey. Acta Biochim. Pol. 48:599-610.

43.Zhu, Z., A.M. Friedman, and S.Y. Xu. 2003. Isolation of BsoBI restriction endonuclease variants with altered substrate specificity. J. Mol. Biol. 330:359-372.

44.Fomenkov, A., J.P. Xiao, D. Dila, E. Raleigh, and S.Y. Xu. 1994. The "endo-blue method" for direct cloning of restriction endonuclease genes in E. coli. Nucleic Acids Res. 22:23992403.

45.Bolivar, F., R.L. Rodriguez, P.J. Greene, M.C. Betlach, H.L. Heyneker, H.W. Boyer, J.H. Crosa, and S. Falkow. 1977. Construction and characterization of new cloning vehicles. II. A multipurpose cloning system. Gene 2:95-113.

46.Bullock, W.O., J.M. Fernandez, and J.M. Short. 1987. XL1-Blue: a high efficiency plasmid transforming recA Escherichia coli with beta galactosidase selection. BioTechniques 5:376-379.

47.Sambrook, J., E.F. Fritsch, and T. Maniatis. 1989. Molecular Cloning: A Laboratory Manual, 2nd Ed. CSH Laboratory Press, Cold Spring Harbor, NY.

48.Sanger, F., S. Nicklen, and A.R. Coulson. 1977. DNA sequencing with chain-terminating inhibitors. Proc. Natl. Acad. Sci. USA 74:5463-5467.

49.Biondi, R.M., P.J. Baehler, C.D. Reymond, and M. Veron. 1998. Random insertion of GFP into the cAMP-dependent protein kinase regulatory subunit from Dictyostelium discoideum. Nucleic Acids Res. 26:4946-4952.

50.Herrera, J.E. and J.B. Chaires. 1994 Characterization of preferred deoxyribonuclease I cleavage sites. J. Mol. Biol. 236:405-411.

51.Ostermeier, M., A.E. Nixon, J.H. Shim, and S.J. Benkovic. 1999. Combinatorial protein engineering by incremental truncation. Proc. Natl. Acad. Sci. USA 96:3562-3567.

52.Posfai, G., S.C. Kim, L. Szilak, A. Kovacs, and P. Venetianer. 1991. Complementation by detached parts of GGCC-specific DNA methyltransferases. Nucleic Acids Res. 19:48434847.

Received 11 February 2005; accepted 13 April 2005.

Address correspondence to:

Arvydas Lubys

Fermentas UAB, Graičiūno 8

2028 Vilnius, Lithuania

e-mail:lubys@fermentas.lt

To purchase reprints

of this article, contact

apfeffer@BioTechniques.com 